\title{
BMJ Open Current status of health systems financing and oversight for end-stage kidney disease care: a cross-sectional global survey
}

Emily Yeung (D) , ${ }^{1}$ AK Bello, ${ }^{2}$ Adeera Levin, ${ }^{3}$ Meaghan Lunney (D) , ${ }^{4}$ Mohamed A Osman, ${ }^{5}$ Feng Ye, ${ }^{2}$ Gloria Ashuntantang, ${ }^{6}$ Ezequiel Bellorin-Font, ${ }^{7}$ Mohammed Benghanem Gharbi, ${ }^{8}$ Sara Davison (1) ,9 Mohammad Ghnaimat, ${ }^{10}$ Paul Harden, ${ }^{11}$ Vivekanand Jha, ${ }^{12}$ Kamyar Kalantar-Zadeh, ${ }^{13}$ Peter Kerr, ${ }^{14,15}$ Scott Klarenbach, ${ }^{5}$ Csaba Kovesdy, ${ }^{16}$ Valerie Luyckx, ${ }^{17}$ Brendon Neuen, ${ }^{18}$ Donal O'Donoghue, ${ }^{19,20}$ Shahrzad Ossareh, ${ }^{21}$ Jeffrey Perl, ${ }^{22,23}$ Harun Ur Rashid, ${ }^{24}$ Eric Rondeau, ${ }^{25}$ Emily See, ${ }^{26}$ Syed Saad, ${ }^{2}$ Laura Sola, ${ }^{27}$ Irma Tchokhonelidze, ${ }^{28}$ Vladimir Tesar, ${ }^{29}$ Kriang Tungsanga, ${ }^{30,31}$ Rumeyza Turan Kazancioglu, ${ }^{32}$ Angela Yee-Moon Wang, ${ }^{33}$ Natasha Wiebe, ${ }^{34}$ Chih-Wei Yang, ${ }^{35}$ Alexander Zemchenkov, ${ }^{36}$ Minhui Zhao, ${ }^{37}$ Kitty J Jager, ${ }^{38}$ Fergus Caskey, ${ }^{39}$ Vlado Perkovic, ${ }^{18}$ Kailash Jindal, ${ }^{5}$ Ikechi G Okpechi (D), ," Marcello Tonelli (D) , ${ }^{41}$ John Feehally, ${ }^{42}$ David CH Harris, ${ }^{43}$ David Johnson ${ }^{44,45}$

To cite: Yeung E, Bello AK, Levin A, et al. Current status of health systems financing and oversight for end-stage kidney disease care: a cross-sectional global survey. BMJ Open 2021;11:e047245. doi:10.1136/ bmjopen-2020-047245

- Prepublication history and additional supplemental material for this paper are available online. To view these files, please visit the journal online (http://dx.doi.org/10.1136/ bmjopen-2020-047245).

Received 24 November 2020 Accepted 08 June 2021

Check for updates

(C) Author(s) (or their employer(s)) 2021. Re-use permitted under CC BY-NC. No commercial re-use. See rights and permissions. Published by BMJ.

For numbered affiliations see end of article.

Correspondence to

Dr Emily Yeung;

emily.yeung@monashhealth.org

\section{ABSTRACT}

Objectives The Global Kidney Health Atlas (GKHA) is a multinational, cross-sectional survey designed to assess the current capacity for kidney care across all world regions. The 2017 GKHA involved 125 countries and identified significant gaps in oversight, funding and infrastructure to support care for patients with kidney disease, especially in lower-middle-income countries. Here, we report results from the survey for the second iteration of the GKHA conducted in 2018, which included specific questions about health financing and oversight of end-stage kidney disease (ESKD) care worldwide.

Setting A cross-sectional global survey.

Participants Key stakeholders from 182 countries were invited to participate. Of those, stakeholders from 160 countries participated and were included.

Primary outcomes Primary outcomes included cost of kidney replacement therapy (KRT), funding for dialysis and transplantation, funding for conservative kidney management, extent of universal health coverage, outof-pocket costs for KRT, within-country variability in ESKD care delivery and oversight systems for ESKD care. Outcomes were determined from a combination of desk research and input from key stakeholders in participating countries.

Results 160 countries (covering $98 \%$ of the world's population) responded to the survey. Economic factors were identified as the top barrier to optimal ESKD care in 99 countries (64\%). Full public funding for KRT was more common than for conservative kidney management (43\% vs $28 \%$ ). Among countries that provided at least some public coverage for KRT, 75\% covered all citizens. Withincountry variation in ESKD care delivery was reported in $40 \%$ of countries. Oversight of ESKD care was present in

\section{Strengths and limitations of this study}

- The study included 160 countries (covering $98 \%$ of the world's population), which provided a comprehensive overview of the state of kidney care around the world.

- The study combined findings from desk research as well as survey responses from on-the-ground stakeholders to provide a holistic perspective of kidney care in participating countries.

- The 2019 report was the second iteration of the Global Kidney Health Atlas and allowed for monitoring of progress made since the previous report and identification of new and ongoing gaps.

- Data available from low-income countries were limited compared to middle-income and high-income countries, which led to challenges in identifying specific gaps in care provision in nations where this was greatly needed.

all high-income countries but was absent in $13 \%$ of lowincome, $3 \%$ of lower-middle-income, and $10 \%$ of uppermiddle-income countries.

Conclusion Significant gaps and variability exist in the public funding and oversight of ESKD care in many countries, particularly for those in low-income and lowermiddle-income countries.

\section{INTRODUCTION}

The prevalence of kidney disease is increasing rapidly, accounting for 1.2 million deaths in 2015 -an increase of $32 \%$ since $2005 .^{12}$ 
Kidney disease is roughly twice as prevalent as diabetes and 20 times more prevalent than cancer or HIV/AIDS. ${ }^{3}$ In 2013, chronic kidney disease (CKD) was one of the top 10 causes of years of life lost and/or disability adjusted life years in 51 countries. ${ }^{4}$ Of the estimated $4.9-9.7$ million people in need of kidney replacement therapy (KRT) for end-stage kidney disease (ESKD) in 2010, only 2.6 million had access. ${ }^{5}$ The number of people requiring KRT is projected to increase to 14.5 million by 2030 , with only 5.4 million people likely to have access if current trends continue. Only $4 \%$ of people in low-income and middleincome countries who require KRT have access, compared with $60 \%$ of people in high-income countries. ${ }^{5}$

The global burden of kidney disease can be reduced by access to universal health coverage (UHC), which is the cornerstone of health equity. All 192 WHO member states committed to implementing UHC in 2005 and recently recommitted to achieving this goal by 2030 at the UN General Assembly High-Level Meeting on Universal Health Coverage. ${ }^{6}$ Successful delivery of UHC requires adequate health financing and effective leadership and governance, ${ }^{7}$ which have been identified by the WHO as two of the six building blocks necessary for a functioning health system. ${ }^{8}$ Poor governance practices, such as inadequate resource allocation and unstructured care provision, are closely correlated with increased prevalence of ESKD. ${ }^{9}$ Within individual countries, those who are most vulnerable or disadvantaged are at higher risk of incident CKD and rapid disease progression. ${ }^{1}$

The Global Kidney Health Atlas (GKHA) is a multinational, cross-sectional survey designed to assess the current capacity for kidney care across all world regions. The 2017 GKHA involved 125 countries and identified significant gaps in oversight, funding and infrastructure to support care for patients with kidney disease, especially in lowermiddle-income countries. ${ }^{2}$ The report found that roughly half of countries worldwide provided public funding for acute kidney injury (AKI) treatment (56\%), non-dialysis CKD treatment (40\%), dialysis (63\%) and transplantation $(57 \%) .{ }^{2}$ Funding was less common in lower-income countries, with countries in Africa and Southeast Asia being more likely to rely on private funding and out-of-pocket expenditure. ${ }^{2}$ Some countries in Africa and the Middle East reported no organised healthcare system. ${ }^{2}$ Perceived quality of healthcare infrastructure for AKI and CKD care was poor in $40 \%$ of low-income countries, particularly in Africa. ${ }^{2}$ Here, we report results from the survey for the second iteration of the GKHA conducted in 2018, which included specific questions about health financing and oversight of ESKD care worldwide. Oversight has been defined as the body responsible for ensuring effective delivery of kidney care.

\section{METHODS}

Two data collection methods were used for the second iteration of the GKHA: desk research (review of available literature) and an online survey of key stakeholders from each country. Details about the design, validation and distribution of the online survey, data analysis techniques and original questionnaire have been reported elsewhere. ${ }^{1011}$ The project was funded by the International Society of Nephrology (ISN), and all survey participants provided informed consent.

\section{Desk research}

Data sources included literature reviews, databases, the WHO Global Health Observatory, surveys, interviews and the WHO Non-Communicable Disease Strategy. A scoping review of published articles and grey literature was also performed to extract estimates of the annual costs of maintenance haemodialysis (HD), maintenance peritoneal dialysis (PD) and kidney transplantation in the first year and subsequent years and of the cost ratio of maintenance HD to PD. Since included studies were from different years and from countries with different currencies, reported costs were converted to international dollars (2016 US\$) using standard techniques (online supplemental figure 1).

\section{Country survey}

A project leader from each of the 10 ISN regions identified three key stakeholders (a nephrology society leader, a leader of a consumer representative organisation and a policymaker) for each country in his or her region (182 countries total). The stakeholders were subsequently invited by email to participate in the survey (available in English, French and Spanish) via an online portal (www. redcapcloud.com) between July and September 2018. The survey data were used to provide information on funding for KRT and conservative kidney management, UHC, out-of-pocket costs for KRT, within-country variations in ESKD care delivery and oversight of ESKD care.

\section{Analysis}

Analysis was performed using a statistical analysis framework (Assessing National Capacity for the Prevention and Control of Non-Communicable Diseases) developed by the WHO. World Bank income categories based on gross national income per capita in 2018 were used to classify countries as low-income, lower-middle-income, uppermiddle-income and high-income countries. ${ }^{12}{ }^{13}$ Results were analysed using a descriptive statistical approach and stratified by World Bank income group and ISN region. An emphasis was placed on identifying key gaps and challenges across domains based on a pre-existing protocol. ${ }^{11}{ }^{14}$ Reporting followed the Guidelines for Accurate and Transparent Health Estimates Reporting statement ${ }^{12}$ and the Checklist for Reporting Results of Internet E-Surveys. ${ }^{13}$ The analyses were performed using Stata V.15 software (Stata Corporation, 2017).

\section{RESULTS}

Among the 182 countries surveyed, 160 responded, accounting for over $98 \%$ of the world's population. 
Annual Cost of KRT Modalities by World Bank Income Group

70,000

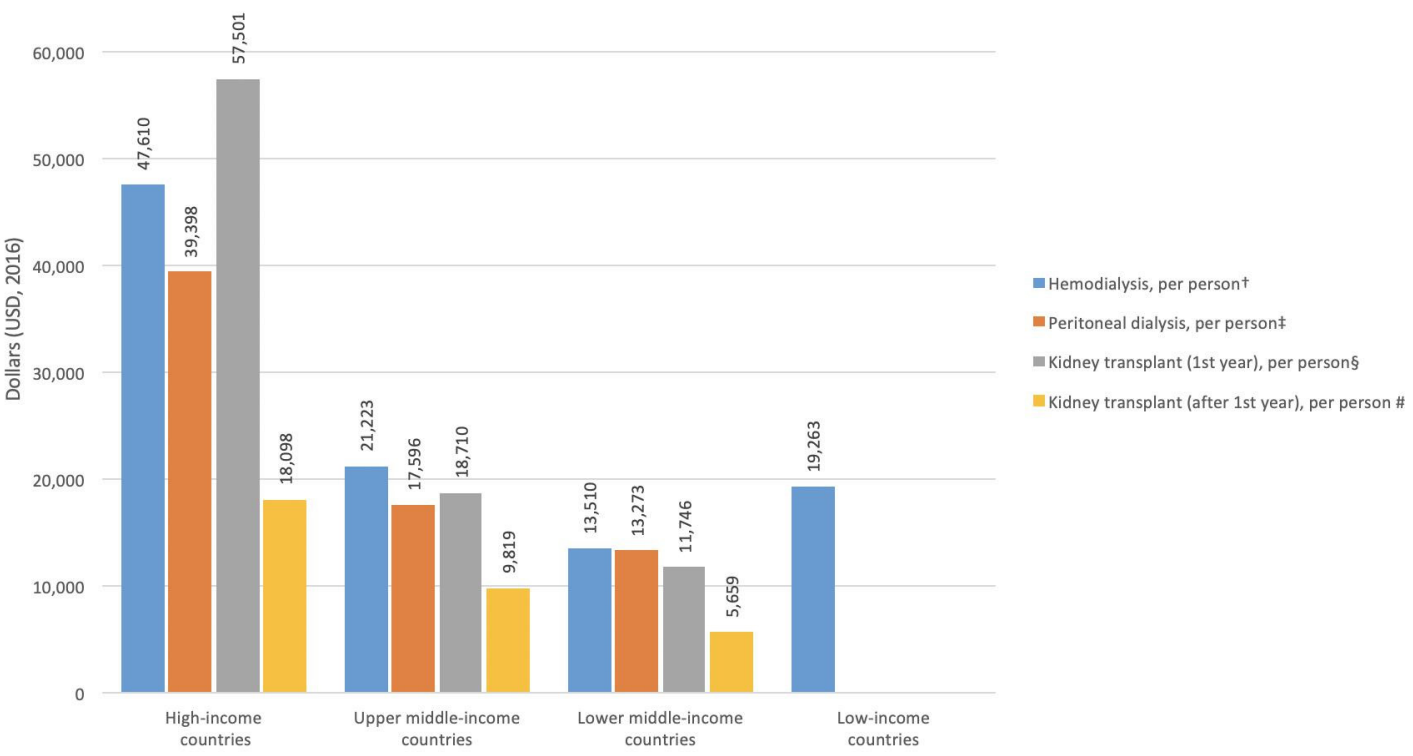

Figure 1 Annual cost of kidney replacement therapy (KRT) modalities by World Bank income group.

Economic factors were identified as the top barrier to optimal ESKD care $(64 \%)$, followed by patient knowledge and attitude (63\%); nephrologist availability (60\%); physician availability, access, knowledge and/or attitude (58\%); capability of the healthcare system (55\%); and distance from care or prolonged travel time $(55 \%)$.

\section{Cost of KRT}

The annual cost of KRT (measured as 2016 US\$) was highest in high-income countries and decreased as World Bank income group decreased (figure 1 and online supplemental table 1). In high-income countries, HD incurred higher costs per year than PD. Kidney transplantation (first year) was costlier than either dialysis modality, but cost savings were significant in each subsequent year of post-transplant care. This trend continued in uppermiddle-income countries, with the notable exception that kidney transplantation (first year) was less costly than dialysis. In lower-middle-income countries, the costs of HD and PD were comparable, with kidney transplantation (first year) being less costly than dialysis. Cost estimates were only available for HD in low-income countries (figure 1 and online supplemental table 1).

Comparing costs of HD to PD, HD was more costly than PD in the majority of high-income and upper-middleincome countries, whereas PD was more costly than HD in the majority of lower-middle-income countries. Unfortunately, insufficient data were available from low-income countries to compare costs (figure 2).

\section{Funding for dialysis and transplantation}

The average number of people receiving treatment for ESKD was 759 per million population (pmp) worldwide, with higher rates of treatment in high-income countries (969 pmp) compared with upper-middle-income (550.2 pmp), lower-middle-income (321 pmp) and low-income countries (4.4 pmp), despite similar rates of ESKD incidence in high-income (149 pmp), upper-middle-income (126 pmp) and lower-middle-income countries (129.9 pmp).

Globally, public funding for KRT with no fees at point of delivery was available in $43 \%$ of countries, with an additional $21 \%$ offering public funding with some fees at the point of delivery (figure 3). Public funding for KRT was more prevalent in high-income countries $(78 \%)$, with the highest rates of private and out-ofpocket funding in low-income countries $(17 \%)$. Countries in the Middle East had the highest availability of full or partial public funding for KRT $(100 \%)$, followed by Western Europe $(91 \%)$, the Newly Independent States (NIS) and Russia (89\%), North and East Asia (85\%) and Eastern and Central Europe (84\%) (figure 3). Medications for KRT patients were free at the point of delivery in $62 \%$ of countries overall, with an additional $21 \%$ offering public funding with some fees at the point of delivery. Medications were most likely to be fully covered in Eastern and Central Europe (95\%), the Middle East (73\%) and Western Europe (45\%), with the lowest rates of coverage in North America and the Caribbean (11\%), Africa (23\%) and Latin America (28\%). The proportion of countries providing full coverage for medications was much lower for lowincome $(19 \%)$ and lower-middle-income $(23 \%)$ countries, compared with upper-middle-income (54\%) and high-income $(50 \%)$ countries (online supplemental table 2).

Medications for patients on KRT were covered in the minority $(41 \%)$ of countries (online supplemental table $2)$. The majority of countries $(58 \%)$ provided some or 
HD to PD Cost Ratio: High Income Countries

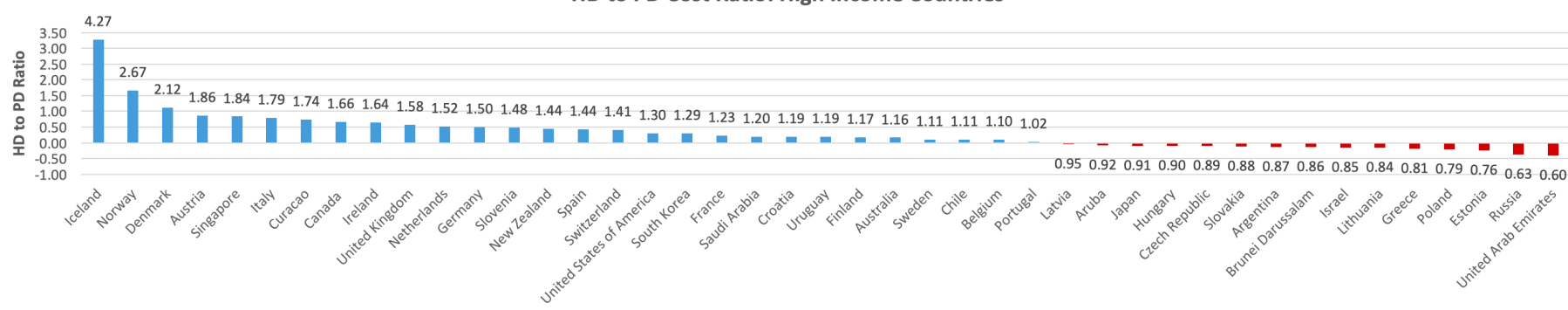

full public funding for the creation of vascular access. Central venous catheters were covered in $58 \%$ of countries, and arteriovenous fistula or graft creation was covered in $54 \%$ of countries (online supplemental tables 3 and 4). Access creation for PD was publicly funded in $54 \%$ of countries (online supplemental table 5). Transplantation surgery was covered by full or partial public funding in 53\% of countries. Coverage increased with World Bank income level (low, $50 \%$; lower middle, $74 \%$; upper middle, $85 \%$; and high, $98 \%$ ). Countries in Eastern and Central Europe, the Middle East, the NIS and Russia, North and East Asia and Western Europe had the highest coverage for all surgical aspects of kidney care, including both dialysis access creation and kidney transplantation. Coverage for all aspects of KRT was provided by $75 \%$ of high-income, $43 \%$ of upper-middle-income, $19 \%$ of lower-middle-income and $0 \%$ of low-income countries (online supplemental figure 2).

\section{Funding for conservative kidney management}

Overall, public funding for KRT was more common than funding for conservative kidney management (online supplemental figure 2). Globally, 28\% of countries provided full public funding for conservative kidney management, with an additional 20\% charging some fees at point of delivery (online supplemental table 6). Conservative kidney management was more commonly excluded from public funding in South Asia, the NIS and Russia, Africa, Oceania and South East Asia (OSEA) and Latin America. Management of ESKD complications (ie,

Funding Models for KRT

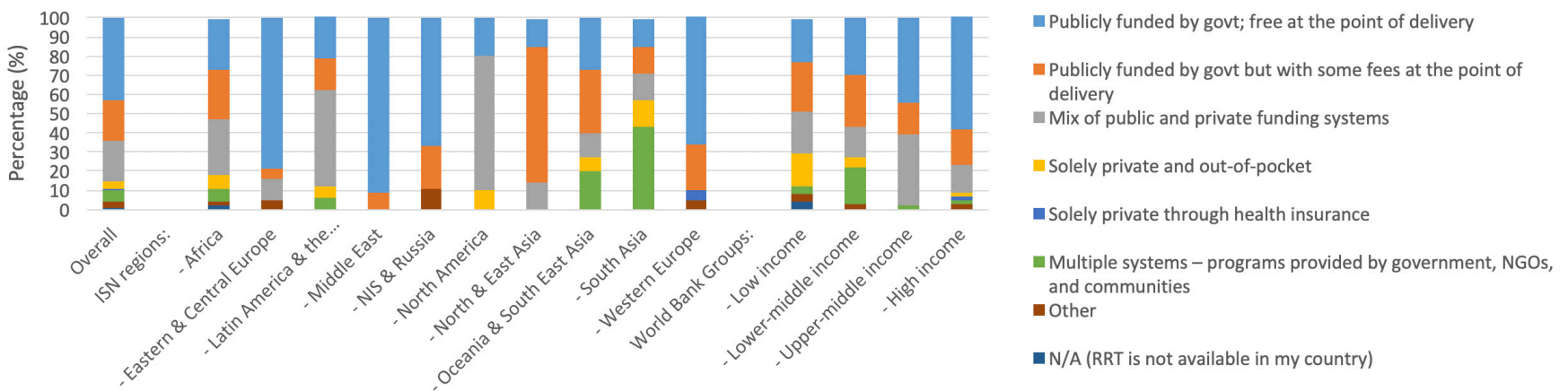

Figure 3 Funding models for kidney replacement therapy (KRT). NGOs, non-governmental organisations; KRT, rapid transformational therapy. 
Extent of Universal Coverage for KRT

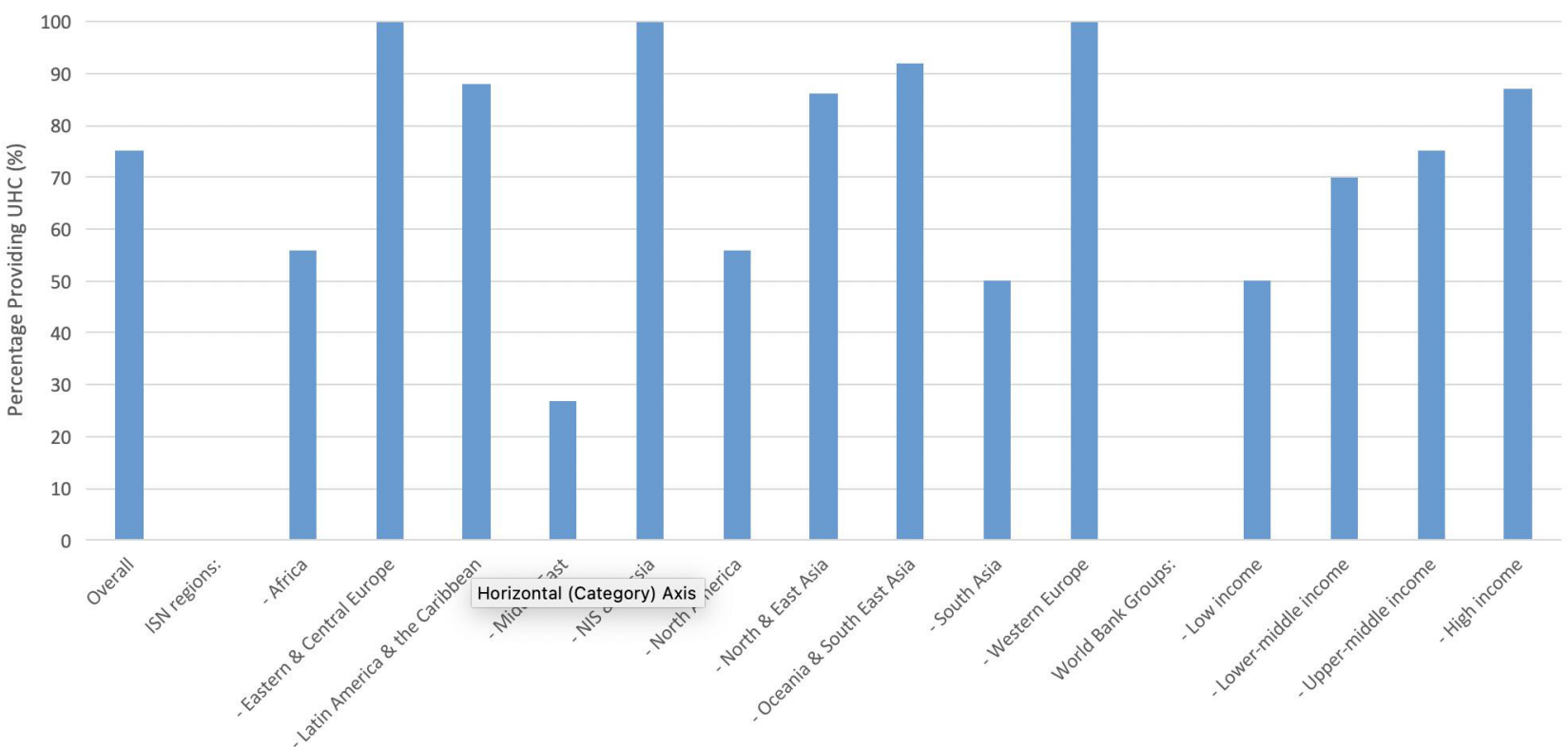

Figure 4 Extent of universal coverage for kidney replacement therapy (KRT). UHC, universal health coverage.

anaemia, bone disease, and malnutrition) was more likely to be excluded from coverage in South Asia, the NIS and Russia, Africa and North America.

\section{Universal health coverage}

Among the countries that provided at least some public funding for KRT, $75 \%$ provided coverage to all citizens. However, the level of UHC for KRT varied significantly by region, with predictably higher rates with increasing country income level. UHC for KRT was provided in Eastern and Central Europe, the NIS and Russia and Western Europe, with the lowest rates of UHC provided in the Middle East (27\%), followed by South Asia (50\%), North America (56\%), and Africa (56\%) (figure 4).

\section{Out-of-pocket costs for KRT}

Globally, $8 \%$ of patients were required to pay the entirety of HD costs, while $27 \%$ of patients did not pay any outof-pocket costs. Similarly, for PD, patients in 5\% of countries paid $100 \%$ of costs (Albania, Botswana, Bangladesh, Democratic Republic of Congo, Croatia, Kosovo, Moldova and New Caledonia), while 23\% of patients paid no fees. For transplantation, patients in $9 \%$ of countries paid $100 \%$ of costs (Albania, Croatia, Ghana, Haiti, Jordan, Moldova, Nigeria, Saudi Arabia, Syrian Arab Republic and the United Arab Emirates), while patients in $31 \%$ of countries paid nothing (online supplemental figure 2).

In general, residents of lower-income-countries had a higher likelihood of bearing the full burden of HD costs. Patients paid $100 \%$ of HD costs in $14 \%$ of low-income countries, compared with $11 \%$ of lower-middle-income, $7 \%$ of upper-middle-income and $4 \%$ of high-income countries. Conversely, patients paid no out-of-pocket costs for HD in $39 \%$ of high-income countries, compared with $20 \%$ of upper-middle-income, $17 \%$ of lower-middleincome and $23 \%$ of low-income countries. Globally, a small proportion of patients paid $100 \%$ of costs for PD, regardless of the country's income level (low, $5 \%$; lower middle, 9\%; upper middle, 5\%; and high, 4\%). Full funding for PD followed a pattern similar to that for $\mathrm{HD}$, with patients in $0 \%$ of low-income, $14 \%$ of lowermiddle-income, $24 \%$ of upper-middle-income and $36 \%$ of high-income countries paying no fees. Very few lowincome countries reported on the affordability of kidney transplantation, with information available for only five countries (Cambodia, Burundi, Democratic Republic of Congo, Nepal and Tanzania). Patients bore the full costs of transplantation in $20 \%$ of low-income, $17 \%$ of lowermiddle-income, $6 \%$ of upper-middle-income and $6 \%$ of high-income countries. Full coverage for transplantation was available for patients in $20 \%$ of low-income, $17 \%$ of lower-middle-income, $24 \%$ of upper-middle-income and $44 \%$ of high-income countries (figure 5 ).

\section{Within-country variability in ESKD care delivery}

Intracountry variation in the delivery of ESKD care was reported in $40 \%$ of countries, with the highest variation in OSEA, Africa, South Asia and North America. Intracountry variation was highest in low-income $(65 \%)$ and upper-middle-income (64\%) countries, slightly lower in lower-middle-income countries $(46 \%)$ and much lower in high-income countries (18\%). In addition, variability between children and adults in care delivery and access to KRT increased as country income level decreased.

\section{Oversight of ESKD care}

All high-income countries reported the existence of ESKD management and oversight systems; however, 13\% of low-income, $3 \%$ of lower-middle-income and $10 \%$ of upper-middle-income countries lacked such systems. The majority of oversight took place at the national level $(56 \%)$; the hospital, trust or organisational level (38\%); 

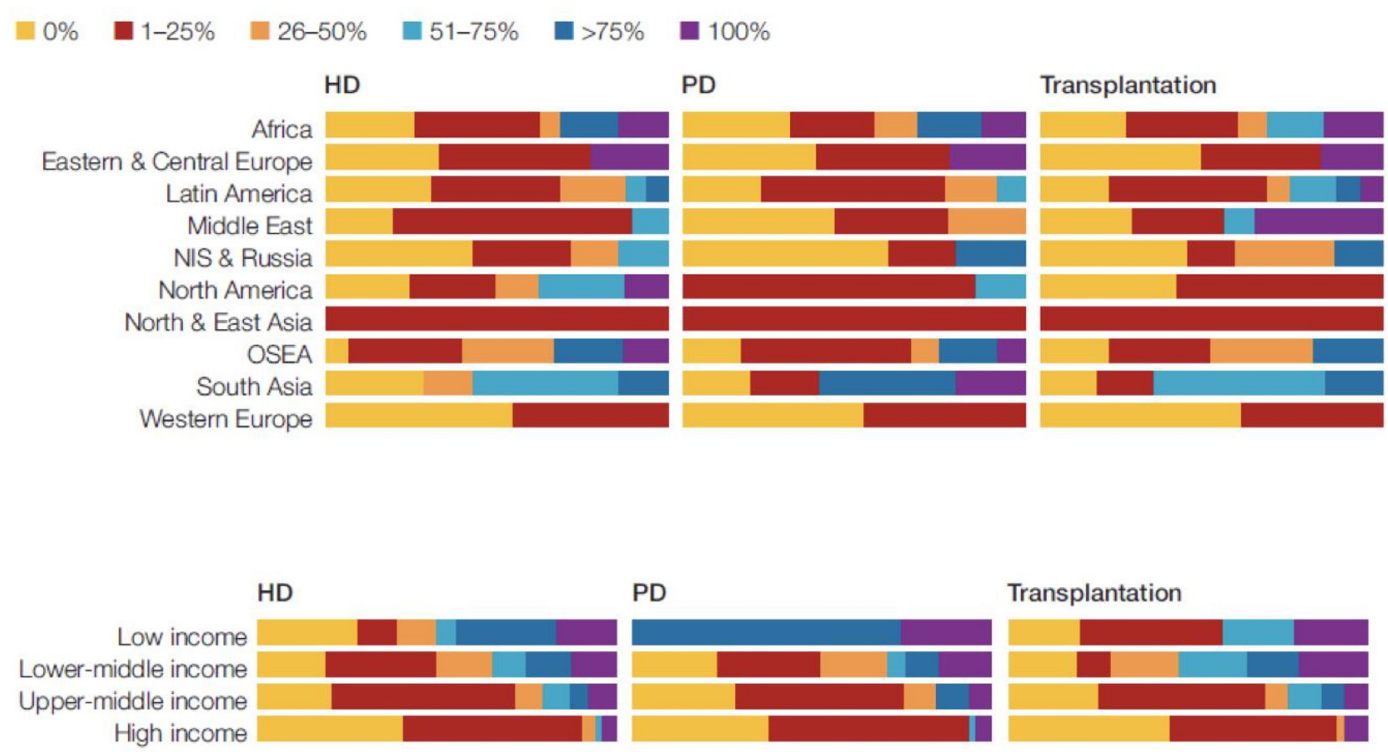

Figure 5 Proportion of treatment costs paid directly by patients, International Society of Nephrology region and World Bank income group. HD, haemodialysis; OSEA, Oceania and South East Asia; PD, peritoneal dialysis.

or the provincial, regional or state level (21\%) (online supplemental table 7).

\section{DISCUSSION}

Full public funding for both conservative kidney management and KRT decreased as World Bank income level decreased. Lower country income level was also associated with poorer health system oversight and higher levels of intracountry variability in care delivery.

UHC is defined by the WHO as 'access to necessary promotive, preventive, curative and rehabilitative health interventions for all at an affordable cost'. The aim of UHC is to avoid catastrophic out-of-pocket expenses for access to health services, defined as healthcare expenditures exceeding $10 \%$ of household income. ${ }^{14}$ Financial protection is lacking globally and disproportionately affects those who are most vulnerable: the poor, minority populations, rural populations and women and children. ${ }^{14}$ Unfortunately, this study revealed that countries in the Middle East, South Asia, North America and Africa did not provide UHC for KRT. Globally, a significant proportion of people living with ESKD paid for treatment entirely out of pocket.

In lower-middle-income countries where $100 \%$ of costs are out of pocket, a single month of essential medicines for kidney care can cost up to 18 days' wages. ${ }^{1}$ Such outof-pocket costs relative to wages are even more stark for expensive kidney care treatments, such as immunosuppressive medications and dialysis consumables. A study of Organisation for Economic Co-operation and Development countries demonstrated an association between higher drug costs and out-of-pocket expenses, with poorer medication compliance among dialysis patients. ${ }^{15}$ In India, a study demonstrated higher adherence to dialysis three times per week when external financial support was provided and high rates of dialysis cessation $(60 \%)$ in the absence of support. ${ }^{15}$ In sub-Saharan Africa, over $95 \%$ of patients with ESKD were unable to access dialysis due to financial and other barriers. Among those with access, only $10 \%$ of adults remained on dialysis beyond 3 months, resulting in high mortality. ${ }^{16}$ The majority of attrition occurred within 2 weeks of dialysis commencement due to an inability to pay the mean cost of US\$100-150 per session. ${ }^{16}$ Lack of UHC for ESKD treatment inevitably leads to out-of-pocket costs and adverse outcomes. Coverage for transplantation without coverage for immunosuppressant medications, antihypertensives or vaccinations, for example, resulted in suboptimal outcomes and reduced the cost-effectiveness of the upfront investment in the transplantation process. ${ }^{17}$ Ultimately, the decision to provide funding for healthcare is a reflection of a country's priorities. Despite a per capita income of only US $\$ 400$, citizens in Rwanda enjoy access to basic health services, with the country allocating $19.5 \%$ of its total annual spending to healthcare. There are 182 other WHO member states with comparable or higher GDPs that are much farther away from achieving UHC. ${ }^{18}$

Conservative kidney management is important, given resource constraints (both patient and system related) in many countries that limit access to KRT. ${ }^{9}$ In this study, only a minority of countries provided full public funding for conservative kidney management, with some regions excluding it from public funding altogether. Choicerestricted conservative kidney management can be provided in settings where KRT is not an option due to insufficient resources. ${ }^{17}$ Despite a lack of choice, conservative kidney management is certainly preferable to a complete lack of care. Interventions should focus on delaying disease progression, minimising adverse effects and complications, managing active symptoms, providing 
psychosocial support for patients and their families and promoting shared decision making in all domains of care. ${ }^{17}$

In the majority of developing countries, only $5 \%$ of those with ESKD as a complication of diabetes or hypertension can access KRT, leaving millions without care. ${ }^{19}$ Widespread access to KRT is unlikely to be established quickly, particularly in low-income and lower-middleincome countries, and the minimisation of suffering and maximisation of quality of life should be prioritised. Alongside conservative kidney management, the role of preventative health should not be overlooked as a cost-effective intervention. Rates for hypertension and diabetes are projected to increase by $80 \%-100 \%$ in developing countries in the coming two decades and by $20 \%-50 \%$ in developed countries. At present, $25 \%-45 \%$ of patients receiving KRT in developing countries have ESKD secondary to one of these conditions. ${ }^{19}$ Thus, there is significant potential for early intervention to prevent the onset and progression of CKD.

Economic factors were identified as top barriers to optimal ESKD care globally. Perhaps unsurprisingly, only $28 \%$ of countries provided full public funding for conservative kidney management, and $43 \%$ provided full coverage of all KRT modalities. An adequate health financing system is defined by the WHO as one that raises adequate funds for health, ensures people can use needed services while being protected from financial catastrophe or impoverishment and provides incentives for healthcare providers and users to be efficient. ${ }^{8}$

Four strategies identified to increase healthcare funding are improving the efficiency of taxation, reprioritising government budgets towards health, evaluating innovative financing mechanisms and increasing development assistance for health. ${ }^{6}$ However, it is also important to acknowledge that scaling up of services does not always require increased investment; often, more can be done with existing resources by appropriately matching care provision with the burden of disease, properly distributing healthcare providers, improving the organisation of primary care, reducing disparities in health access and ensuring interventions are evidence based and cost effective. ${ }^{9}$ Major sources of healthcare inefficiency identified by the WHO include an inefficient or inappropriate mix of funding between prevention and treatment, a lack of guidelines to maintain clinical care standards and weak procurement strategies that lead to the underuse of generic medications and overuse of substandard or counterfeit medicines. ${ }^{18}$

Ultimately, no country is able to provide coverage for all available life-prolonging therapies for all citizens. Making decisions about which therapies to cover is critically important to maximise impact, with ongoing evaluation to keep pace with changes in resource availability. A framework exists for low-income and middle-income country governments to use when considering establishment or scaling up of programmes for prevention and management of kidney failure. This framework prioritises preventative care due to high cost efficiency and potential for integration into other non-communicable disease prevention programme. ${ }^{20}$ Alongside prevention, conservative care programmes are recommended. Within KRT, kidney transplantation is the most cost effective; however, this may be limited by lack of surgical expertise or lack of appropriate safeguards to facilitate organ donation and prevent organ trafficking. In most contexts, PD is equally effective but more cost effective in the majority of lowresource settings compared with $\mathrm{HD}$, but local variation may exist depending on cost and local supply of PD fluid. ${ }^{20}$ When UHC was first introduced in Thailand in 2002, a limit of 100000 baht per quality-adjusted life year was set by the National Health Security Office. This meant that although prescription medicines, ambulatory care, hospitalisation, disease prevention and health promotion were offered free of charge, KRT, which costed 400000 baht per patient per year, was not. When resources increased after the country recovered from the Asian economic crisis, the scheme was subsequently expanded in 2008 to include KRT. ${ }^{1821}$ Health systems in all countries will face increasing financial strain in providing adequate care for an increasing global burden of CKD. It is therefore critically important that countries make ongoing financial commitments to provide equitable health coverage and UHC and use available resources efficiently.

In addition to appropriate health financing, appropriate health governance involving both oversight and priority setting, as well as subsequent evaluation of progress and outcomes, is crucial for optimal ESKD care. Developing effective policy requires defining problems, considering policy alternatives and evaluating costs versus benefits to create action plans that accurately inform health financing decisions and subsequent quantitative and qualitative evaluations of outcomes. ${ }^{9}$ With the exception of high-income countries, a significant proportion of countries currently lack ESKD management systems and oversight.

Informed priority setting requires identifying locally relevant risk factors and developing policies and plans that address the unique needs of the population. ${ }^{1}$ For example, Mesoamerican, Balkan and Chinese herbal nephropathies are associated with region-specific risk factors. ${ }^{9}$ Effective policy also plays a pivotal role in disease prevention. Aggressive tobacco taxation has led to a reduction in smoking prevalence and has been shown in some countries to reduce ethnic inequalities in smoking behaviours; typically, higher smoking rates are observed in minority groups. ${ }^{22}$ Similarly, taxation of sugary beverages in Mexico has reduced accessibility to and the appeal of unhealthy foods and provides an additional revenue stream for health expenditures. ${ }^{1}$ Just over 1 year after the introduction of the tax, sugar-sweetened beverage consumption in Mexico declined by $12 \%$, with the greatest reduction among households with low socioeconomic status, which are associated with disproportionate rates of obesity. ${ }^{23}$ Reduction in unhealthy food consumption at the population level reduces the incidence of 
hypertension, diabetes and obesity; all of which are important and modifiable risk factors for CKD.

Health systems must also place a specific emphasis on the establishment of equitable health access. Despite establishing UHC in 2002, residents of Thailand experienced inequitable access to KRT across three healthcare schemes, with some having no access at all. To combat inequality, Thailand introduced a 'PD first' policy in 2008, which required more dialysis centres to be established and more trained staff to be hired, thereby increasing access to KRT, which has improved both survival and quality of life for residents with ESKD. ${ }^{24}$

Reasonable health expenditure is necessary but insufficient to achieve good health outcomes. The USA, for example, spends $17.8 \%$ of its gross domestic product (GDP) on healthcare-more than the average among all high-income countries $(11.5 \%)$ and more per capita than any other nation. ${ }^{21}$ Despite this, Americans have the lowest life expectancy, highest infant mortality and highest percentage of overweight or obese adults among these countries. ${ }^{25}$ This healthcare paradox is often attributed to prioritising curative treatment over preventative health, social determinants of health and equitable access. ${ }^{26}$ Ineffective oversight leads to suboptimal policy informing the allocation of funds for healthcare, which in turn leads to resource strain and poor health outcomes. This again highlights the importance of prioritising the development of oversight mechanisms in upper-middleincome, lower-middle-income and low-income countries.

Global optimisation of ESKD care requires careful management of limited resources and the establishment and strengthening of health oversight and governance mechanisms to manage competing priorities. The high cost of KRT limits the feasibility of public funding in many countries, particularly in low-resource settings. This makes a focus on ESKD prevention particularly important, along with increasing availability of conservative kidney management. HD tended to be less expensive than PD in lower-middle-income countries. Countries that are looking to establish KRT programmes should carefully evaluate costs versus benefits in a context-specific manner to determine the suitability of HD compared with PD (including consideration of home-based treatment options) and implement appropriate compensatory schemes to encourage its use and provide equitable access. Importantly, this study found that cost estimates were only available for HD in low-income countries, which made it impossible to make such comparisons.

Looking ahead, the increasing prevalence of CKD risk factors (diabetes, hypertension and obesity), rising global temperatures due to climate change, changes in disease epidemiology (ie, increased risk of CKD of unknown aetiology and Central American nephropathy) and a demographical shift towards an ageing population are likely to increase the burden of CKD. Emerging technologies may provide additional tools to tackle CKD. Mobile phones have already significantly enhanced the capacity to spread health information and improve health literacy, as well as reduce barriers, including long travel times to seek medical assistance. ${ }^{27}$ Telemedicine is already being used to increase capacity for and access to kidney care for populations in low-resource settings and for underserved populations in high-income settings. ${ }^{27}$ In the first major dialysis technology breakthrough since its invention in 1943, the world's first affordable dialysis machine was unveiled in 2016 as a competition entry for the Affordable Dialysis Prize. The prize was a global challenge to create a low-cost device (costing less than US\$1000 to manufacture and able to run on less than US $\$ 5$ per day) that is powered by solar energy and can purify any water source. Pilot testing for the device is scheduled to begin in $2020 .^{28}$ If successful, the device has the potential to revolutionise the global delivery of dialysis.

\section{Strengths}

This study included 160 countries (covering $98 \%$ of the world's' population), thereby providing a comprehensive overview of the state of kidney care around the world. The use of a combination of data from desk research and survey responses from on-the-ground stakeholders provided a holistic perspective of kidney care in participating countries. The 2019 report was the second iteration of the GKHA and allowed for monitoring of progress made since the previous report and identification of new and ongoing gaps.

\section{Limitations}

These cross-sectional studies captured a snapshot of kidney care at a particular point in time and may not have been representative of the time period of interest. While the cross-sectional design was well suited to facilitate comparisons of global kidney care between countries and regions, they were offset by a relative lack of granular detail required to guide specific programme and systematic change. Data available from low-income countries were limited compared with middle-income and high-income countries, which led to challenges in identifying specific gaps in care provision in nations where this was greatly needed. This is a well-documented issue in low-resource settings and continues to limit the conclusions that can be drawn regarding the state of health and health systems in these countries. Not all countries had a consumer organisation to represent the patient perspective.

\section{CONCLUSION}

From 2017 to 2019, there have been marginal improvements in health systems oversight; overall, fewer countries have no structured governance. However, economic factors remain a top barrier to ESKD care, with suboptimal rates of UHC, inadequate public funding for both KRT and conservative kidney management and ongoing inequalities in care delivery within countries. The potential for emerging technologies to improve affordability and access to CKD care and to enhance the capacity of health systems, particularly in low-resource settings, is 
promising. However, countries must commit to providing UHC, ensure adequate focus on preventative health, establish conservative kidney management pathways for those without KRT access, improve health financing by increasing healthcare funding and reducing spending inefficiencies and establish strong governance systems that engage in evidence-based priority setting and monitoring to be well positioned for the adoption and implementation of these technologies. This second iteration of the GKHA can be used as a marker of progress in global capacity for the provision of CKD care moving forward.

\section{Author affiliations}

${ }^{1}$ Monash Health, Clayton, Victoria, Australia

${ }^{2}$ Division of Nephrology and Immunology, University of Alberta, Edmonton, Alberta, Canada

${ }^{3}$ Division of Nephrology, University of British Columbia, Vancouver, British Columbia, Canada

${ }^{4}$ Department of Community Health Sciences, University of Calgary, Calgary, Alberta, Canada

${ }^{5}$ Medicine, University of Alberta, Edmonton, Alberta, Canada

${ }^{6}$ Faculty of Medicine and Biomedical Sciences, University of Yaounde I Faculty of

Medicine and Biomedical Sciences, Yaounde, Cameroon

${ }^{7}$ Department of Internal Medicine, Saint Louis University, Saint Louis, Missouri, USA ${ }^{8}$ Urinary Tract Diseases Department, University of Hassan II Casablanca Faculty of Science Ain Chock, Casablanca, Morocco

${ }^{9}$ Division of Nephrology and Immunology, University of Alberta Faculty of Medicine and Dentistry, Edmonton, Alberta, Canada

${ }^{10}$ Department of Internal Medicine, The Specialty Hospital, Amman, Jordan

${ }^{11}$ Oxford Kidney Unit, Oxford University Hospitals NHS Trust, Oxford, UK

${ }^{12}$ George Institute for Global Health, New Dehli, India

${ }^{13}$ Division of Nephrology and Hypertension, University of California Irvine Medical Center, Orange, California, USA

${ }^{14}$ Department of Nephrology, Monash Medical Centre Clayton, Clayton, Victoria, Australia

${ }^{15}$ Department of Medicine, Monash University, Clayton, Victoria, Australia

${ }^{16}$ Nephrology, Memphis VA Medical Center, Memphis, Tennessee, USA

${ }^{17}$ Institute of Biomedical Ethics and the History of Medicine, University of Zurich

Institute of Biomedical Ethics History of Medicine, Zurich, Switzerland

${ }^{18}$ The George Institute for Global Health, Newtown, New South Wales, Australia

${ }^{19}$ Salford Royal Hospitals NHS Trust, Salford, UK

${ }^{20}$ Faculty of Biology Medicine and Health, The University of Manchester, Manchester, UK

${ }^{21}$ Hasheminejad Kidney Center, Iran University of Medical Sciences, Tehran, Iran

${ }^{22}$ Department of Nephrology, St Michael's Hospital, Toronto, Ontario, Canada

${ }^{23}$ Division of Nephrology, University of Toronto Faculty of Medicine, Toronto, Ontario, Canada

${ }^{24}$ Department of Nephrology, Kidney Foundation Hospital and Research Institute, Dhaka, Bangladesh

${ }^{25}$ Intensive Care Nephrology and Transplantation Department, Assistance

Publique-Hopitaux de Paris, Paris, France

${ }^{26}$ Department of Intensive Care, Austin Health, Heidelberg, Victoria, Australia

${ }^{27}$ Dialysis Unit, CASMU-IAMPP, Montevideo, Uruguay

${ }^{28}$ Nephrology Development Clinical Center, Tbilisi State Medical University, Tbilisi, Georgia

${ }^{29}$ Department of Nephrology, Charles University, Praha, Czech Republic

${ }^{30}$ Department of Medicine, King Chulalong Memorial Hospital, Bangkok, Thailand

${ }^{31}$ Bhumirajanagarindra Kidney Institute, Bangkok, Thailand

${ }^{32}$ Division of Nephrology, Bezmialem Vakif Universitesi, Istanbul, Turkey

${ }^{33}$ Department of Medicine, University of Hong Kong, Hong Kong, Hong Kong

${ }^{34}$ Department of Medicine, University of Alberta Faculty of Medicine and Dentistry,

Edmonton, Alberta, Canada

${ }^{35}$ Kidney Research Center, Chang Gung University College of Medicine, Taoyuan, Taiwan

${ }^{36}$ Department of Internal Disease and Nephrology, North-Western State Medical University named after I I Mechnikov, Sankt-Peterburg, Russia
${ }^{37}$ Renal Division, Peking University First Hospital, Peking University Institute of Nephrology, Beijing, China

${ }^{38}$ Department of Medical Informatics, Academic Medical Center, University of Amsterdam, Amsterdam, Netherlands

${ }^{39}$ Population Health Sciences, University of Bristol, Bristol, UK

${ }^{40}$ Medicine, University of Cape Town, Cape Town, South Africa

${ }^{41}$ Department of Medicine, Cumming School of Medicine, University of Calgary,

Calgary, Alberta, Canada

${ }^{42}$ Department of Infection, Inflammation and Immunity, University of Leicester, Leicester, UK

${ }^{43}$ Centre for Transplantation and Renal Research, Westmead Institute for Medical Research, Westmead, New South Wales, Australia

${ }^{44}$ Centre for Kidney Disease Research, The University of Queensland, Saint Lucia, Queensland, Australia

${ }^{45}$ Metro South Integrated Nephrology and Transplant Services (MINTS), Princess Alexandra Hospital, Woolloongabba, Queensland, Australia

Contributors EY contributed to the design of the work, interpreted the data for the work, drafted the work and was involved in the final approval of the version to be published. AKB, AL and DJ contributed to the conception and design of the work, revised it critically and were involved in the final approval of the version to be published. ML, MAO and FY analysed and interpreted the data for the work, revised it critically and were involved in the final approval of the version to be published. AL, GA, EB-F, MG, SD, MBG, PH, VJ, KK-Z, PK, SK, CK, VL, BN, DOD, SO, JP, HUR, ER, ES, SS, LS, IT, VT, KT, RTK, AY-MW, NW, C-WY, AZ, MZ, KJJ, FC, VP, KJ, IGO, MT, JF and $\mathrm{DCHH}$ revised the work critically and were involved in the final approval of the version to be published.

Funding The authors have not declared a specific grant for this research from any funding agency in the public, commercial or not-for-profit sectors.

Competing interests None declared.

Patient and public involvement statement There was no public or patient involvement.

Patient consent for publication Not required.

Ethics approval The project was approved by the University of Alberta Research Ethics Committee (protocol number: PR000063121).

Provenance and peer review Not commissioned; externally peer reviewed.

Data availability statement No data are available. No additional data available.

Supplemental material This content has been supplied by the author(s). It has not been vetted by BMJ Publishing Group Limited (BMJ) and may not have been peer-reviewed. Any opinions or recommendations discussed are solely those of the author(s) and are not endorsed by BMJ. BMJ disclaims all liability and responsibility arising from any reliance placed on the content. Where the content includes any translated material, BMJ does not warrant the accuracy and reliability of the translations (including but not limited to local regulations, clinical guidelines, terminology, drug names and drug dosages), and is not responsible for any error and/or omissions arising from translation and adaptation or otherwise.

Open access This is an open access article distributed in accordance with the Creative Commons Attribution Non Commercial (CC BY-NC 4.0) license, which permits others to distribute, remix, adapt, build upon this work non-commercially, and license their derivative works on different terms, provided the original work is properly cited, appropriate credit is given, any changes made indicated, and the use is non-commercial. See: http://creativecommons.org/licenses/by-nc/4.0/.

\section{ORCID iDs}

Emily Yeung http://orcid.org/0000-0002-1244-1044

Meaghan Lunney http://orcid.org/0000-0003-4954-0529

Sara Davison http://orcid.org/0000-0003-4513-6449

Ikechi G Okpechi http://orcid.org/0000-0002-6545-9715

Marcello Tonelli http://orcid.org/0000-0002-0846-3187

\section{REFERENCES}

1 Luyckx VA, Tonelli M, Stanifer JW. The global burden of kidney disease and the sustainable development goals. Bull World Health Organ 2018;96:414-22.

2 Bello AK, Alrukhaimi M, Ashuntantang GE, et al. Global overview of health systems oversight and financing for kidney care. Kidney Int Supp/ 2018;8:41-51. 
3 Jager KJ, Kovesdy C, Langham R, et al. A single number for advocacy and communication-worldwide more than 850 million individuals have kidney diseases. Kidney Int 2019;96:1048-50.

4 Jager KJ, Fraser SDS. The ascending rank of chronic kidney disease in the global burden of disease study. Nephrol Dial Transplant 2017;32:ii121-8.

5 Liyanage T, Ninomiya T, Jha V, et al. Worldwide access to treatment for end-stage kidney disease: a systematic review. Lancet 2015;385:1975-82.

6 Reeves A, Gourtsoyannis Y, Basu S, et al. Financing universal health coverage-effects of alternative Tax structures on public health systems: cross-national modelling in 89 low-income and middleincome countries. The Lancet 2015;386:274-80.

7 Sachs JD. Achieving universal health coverage in low-income settings. The Lancet 2012;380:944-7.

8 Organization WH. Strengthening health systems to improve health outcomes - WHO's framework for action. Geneva: World Health Organization, 2007.

9 Jha V, Arici M, Collins AJ, et al. Understanding kidney care needs and implementation strategies in low- and middle-income countries: conclusions from a "Kidney Disease: Improving Global Outcomes" (KDIGO) Controversies Conference. Kidney Int 2016;90:1164-74

10 Bello AK, Johnson DW, Feehally J, et al. Global kidney health atlas (GKHA): design and methods. Kidney Int Supp/ 2017;7:145-53.

11 Bello AK, Levin A, Tonelli M, et al. Assessment of global kidney health care status. JAMA 2017;317:1864

12 Stevens GA, Alkema L, Black RE, et al. Guidelines for accurate and transparent health estimates reporting: the gather statement. The Lancet 2016;388:e19-23.

13 Eysenbach G. Improving the quality of web surveys: the checklist for reporting results of Internet E-Surveys (cherries). J Med Internet Res 2004;6:1.

14 Organization WH. Primary health care on the road to universal health coverage: 2019 global monitoring report. Geneva: World Health Organization, 2019.

15 Dodd R, Palagyi A, Guild L, et al. The impact of out-of-pocket costs on treatment commencement and adherence in chronic kidney disease: a systematic review. Health Policy Plan 2018;33:1047-54.
16 Ashuntantang G, Osafo C, Olowu WA, et al. Outcomes in adults and children with end-stage kidney disease requiring dialysis in sub-Saharan Africa: a systematic review. Lancet Glob Health 2017;5:e408-17.

17 Harris DCH, Davies SJ, Finkelstein FO, et al. Increasing access to integrated ESKD care as part of universal health coverage. Kidney Int 2019;95:33

18 Organzation WH. Health systems financing: the path to universal coverage. Geneva, Switzerland: World Health Organization, 2010.

19 Anand S, Bitton A, Gaziano T. The gap between estimated incidence of end-stage renal disease and use of therapy. PLoS One 2013;8:e72860

20 Tonelli M, Nkunu V, Varghese C, et al. Framework for establishing integrated kidney care programs in low- and middle-income countries. Kidney Int Suppl 2020;10:e19-23.

21 Tangcharoensathien V, Witthayapipopsakul W, Panichkriangkrai W, et al. Health systems development in Thailand: a solid platform for successful implementation of universal health coverage. Lancet 2018;391:1205-23.

22 van der Deen FS, Wilson N, Blakely T. A continuation of $10 \%$ annual tobacco tax increases until 2020: Modelling results for smoking prevalence by sex and ethnicity. N Z Med J 2016;129:4.

$23 \mathrm{KDIGO}$. KDIGO 2012 clinical practice guideline for the evaluation and management of chronic kidney disease. Kidney International Supplements 2013;3:150.

24 Liu FX, Gao X, Inglese G, et al. A global overview of the impact of peritoneal dialysis first or favored policies: an opinion. Perit Dial Int 2015;35:406-20.

25 Papanicolas I, Woskie LR, Jha AK. Health care spending in the United States and other high-income countries. JAMA 2018;319:1024

26 Bradley EH, Sipsma H, Taylor LA. American health care paradoxhigh spending on health care and poor health. QJM 2016;110:61-5.

27 Obasola OI, Mabawonku I, Lagunju I. A review of e-health interventions for maternal and child health in Sub-Sahara Africa. Matern Child Health J 2015;19:1813-24.

28 Knight J, Perkovic V. The Affordable dialysis prize steams ahead. The Lancet 2016;387:1040. 